\title{
Systematic review and practice policy statements on urinary tract infection prevention in adults with spina bifida
}

\author{
Michael Tradewell, Joseph J. Pariser, Tony Nimeh, Sean P. Elliott; on behalf of the Neurogenic Bladder \\ Research Group \\ Department of Urology, University of Minnesota, Minneapolis, MN, USA \\ Contributions: (I) Conception and design: All authors; (II) Administrative support: All authors; (III) Provision of study materials or patients: All \\ authors; (IV) Collection and assembly of data: All authors; (V) Data analysis and interpretation: All authors; (VI) Manuscript writing: All authors; (VII) \\ Final approval of manuscript: All authors. \\ Correspondence to: Sean P. Elliott. Department of Urology, University of Minnesota, 420 Delaware St. SE, MMC 394, Minneapolis, MN 55455, USA. \\ Email: selliott@umn.edu.
}

\begin{abstract}
Urinary tract infection (UTI) is a source of morbidity and healthcare costs in adults with spina bifida (ASB). UTI prevention strategies are often recommended, but the evidence of various approaches remains unclear. We performed a systematic review to inform a best practice policy statement for UTI prevention in ASB. On behalf of the Neurogenic Bladder Research Group (NBRG.org), we developed an a priori protocol and searched the published English literature for 30 outcomes questions addressing UTI prevention in ASB. The questions spanned the categories of antibiotics, oral supplements, bladder management factors and social support. Where there was little literature in ASB, we included literature from similar populations with neurogenic bladder (NB). Data was abstracted and then reviewed with recommendations made by consensus of all authors. Level of Evidence $(\mathrm{LoE})$ and Grade of Recommendation (GoR) were according to the Oxford grading system. Of 6,433 articles identified by our search, we included 99 publications. There was sufficient evidence to support use of the following: saline bladder irrigation (LoE 1, GoR B), gentamicin bladder instillation (LoE 3, GoR C), single-use intermittent catheterization (IC) (LoE 2, GoR B), hydrophilic catheters for IC (LoE 2, GoR C), intradetrusor onabotulinumtoxinA injection (LoE 3, GoR C), hyaluronic acid (HA) instillation (LoE 1, GoR B), and care coordination (LoE 3, GoR C). There was sufficient evidence to recommend against use of the following: sterile IC (LoE 1, GoR B), oral antibiotic prophylaxis (LoE 2, GoR B), treatment of asymptomatic bacteriuria (LoE 2, GoR B), cranberry (LoE 2, GoR B), methenamine salts (LoE 1, GoR B), and ascorbic acid (LoE1, GoR B). There was insufficient evidence to make a recommendation for other outcomes. Overall, there are few studies in UTI prevention in the specific population of ASB. Research in populations similar to ASB helps to guide recommendations for UTI prevention in the challenging patient group of ASB. Future studies in UTI prevention specific to ASB are needed and should focus on areas shown to be of benefit in similar populations.
\end{abstract}

Keywords: Neurogenic bladder (NB); spinal dysraphism; antibiotic prophylaxis; bacteriuria; urinary tract infections (UTIs)

Submitted Apr 16, 2018. Accepted for publication Apr 17, 2018.

doi: $10.21037 /$ tau.2018.04.21

View this article at: http://dx.doi.org/10.21037/tau.2018.04.21 


\section{Introduction}

With improved management, more patients with spina bifida are living to adulthood. Urinary tract infection (UTI) remains a source of morbidity in adults with spina bifida (ASB). Compared to matched controls without spina bifida visiting the emergency room, ASB are 9 times more likely to present with UTI (1). Best practices for UTI diagnosis and prevention are difficult to discern for several reasons: $90 \%$ of ASB manage their bladder with intermittent catheterization (IC) and many have undergone surgical reconstruction of their bladder with bowel segments (2). Together, these factors make bacterial urinary colonization ubiquitous. Determining the difference between bacteriuria and UTI is challenging, especially in patients with sensory impairment. Such patients may not sense classic UTI symptoms such as urgency or dysuria. Instead, patients and clinicians are left to interpret vague symptoms such as malaise and lower extremity spasticity in the presence of chronically cloudy, foul-smelling urine. These factors make UTI prevention and antibiotic stewardship challenging. To address this growing need, we performed a systematic review to inform best practice policy statements for UTI prevention in ASB.

\section{Methods}

On behalf of the Neurogenic Bladder Research Group
(NBRG.org), we developed an a priori protocol and searched the English literature for 30 outcomes questions addressing UTI prevention and management in ASB (PROSPERO registration number: CRD42017074931). Pilot searches showed a paucity of literature specific to UTI prevention in ASB. Consequently, our search protocol was expanded to include literature from similar populations with neurogenic bladder (NB) and recurrent UTI; when the literature was still sparse we also searched for relevant evidence in population with recurrent UTI but without NB. These data were captured through three separate database-specific controlled vocabulary and Medical Subject Headings (MeSH) searches within OVID Medline, Embase, and Cochrane databases: the first search captured original research articles on UTI prevention and management in ASB or any NB population; the second search included reviews or guidelines (but not original articles) addressing UTI prevention and management in any patient population. This hierarchical search strategy is illustrated in a Venn diagram (Figure 1).

Abstracts were screened and collated according to the 30 questions grouped into five categories: antibiotics, oral supplements, bladder management factors (e.g., bladder irrigation or detrusor pressure management), IC techniques, and social support. Data abstraction was done independently and in duplicate with a third investigator arbitrating differences. UTI was defined according to the consensus

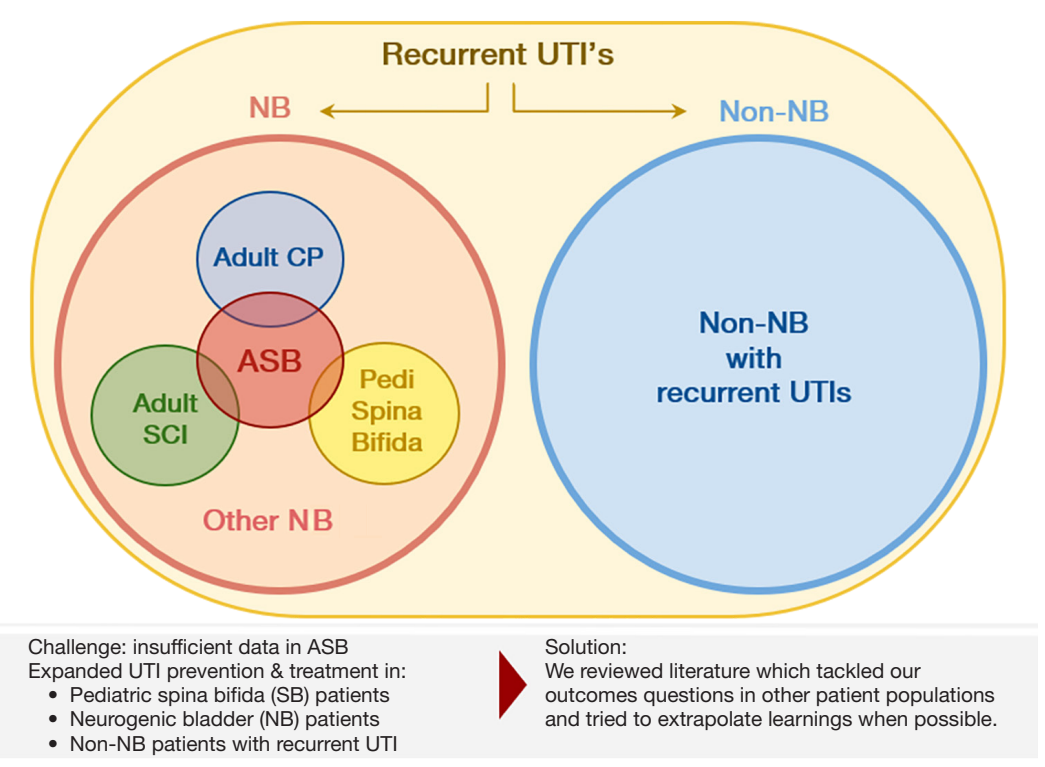

Figure 1 Hierarchical organization of relevant populations reviewed, as they relate to the population of interest: adult spina bifida (ASB). UTI, urinary tract infection. 
definition of the National Institute on Disability and Rehabilitation Research (NIDRR), as $>10^{5}$ colony forming units/mL of a single coliform organism with at least one of the following symptoms: fever, chills, abdominal pain, worsening bladder spasms, new or worsening incontinence, gross hematuria, fatigue, and muscle spasms (3). Bacteriuria was defined as bacteria present in the urine, but not meeting the definition of UTI. In questions with low literature yield, studies assessing asymptomatic bacteriuria rates as the primary outcome were included, these studies are noted herein and were considered less relevant.

The findings of our systematic review were presented to members of the NBRG at its semi-annual research meeting. Individual studies were critiqued, levels of evidence and grades of recommendation were made by consensus of the members. Due to the paucity of data specific to UTI management in ASB and the necessity to include other relevant populations, meta-analysis was not feasible. As such, we constructed a series of practice policy statements based upon data from a systematic review. The degree of relevance of similar populations to ASB was considered in making our final recommendations. Recommendations were made by consensus of all and justification scored according to the Oxford Level of Evidence (LoE) and Grade of Recommendation (GoR) systems (Table 1).

\section{Results}

Of the 6,433 articles identified during initial searches, 2,109 were original articles, 4,310 were reviews, and 14 were guidelines. Of the 2,109 articles, 1,440 were from the Embase database while 669 were from Medline. These articles were reviewed for relevance and duplicates with 2,014 excluded. Ultimately, 95 articles were included in our review (Figure 2).

\section{Recommendations concerning the use of antibiotics for UTI prophylaxis and treatment}

Clinicians should not routinely prescribe long-term prophylactic antibiotics for the prevention of UTI in ASB (LoE 2, GoR B)

\section{Bottom line}

Multiple randomized controlled trials (RCT) have assessed the use of long-term antibiotic prophylaxis in populations with NB. These trials showed heterogeneous results. Positive trials were tempered by increased antimicrobial resistance and adverse events. Secondary literature consistently recommended against the routine use of prophylactic antibiotics though it can be utilized for an individual treatment plan.

\section{Summary of evidence}

Seven RCTs, with mixed results, assess the use of antibiotics for prevention of UTI in patients with NB. Four studies found a significant UTI reduction. The largest was double-blind, placebo-controlled RCT assessing 126 patients in an inpatient setting with recent spinal cord injury (SCI) initiating CIC. This study found fewer UTIs over 16 weeks but had significant adverse events with antibiotic therapy and warned against the risk of resistant organisms (4). Three additional cross-over RCTs supported the use of prophylactic antibiotics in children $(5,6)$ and adults (7) with NB.

Two RCTs that reported on the effect of discontinuing prophylactic antibiotics found no difference in UTI risk with antibiotic prophylaxis in patients with $\mathrm{NB}(8,9)$. A 2005 RCT of 53 children with NB doing CIC on antibiotic prophylaxis found the rate of UTI was increased in the group who continued to use antibiotics compared to those who stopped (10).

Another regimen is weekly oral cyclic antibiotics (WOCA). Two different high dose antibiotics are rotated on a weekly basis. By alternating antibiotics, the risk of resistance may be lessened. Three cohort trials from the same group were identified. Two studies including 38 and 50 patients with NB showed significant reductions in UTI, antibiotics use, and hospitalization with WOCA $(11,12)$. A third limited study assessing WOCA in six pregnant women with NB and recurrent UTI reported a UTI reduction (13).

\section{Clinicians should not perform routine urine culture} surveillance (LoE 2, GoR B) and should not treat asymptomatic bacteriuria for the prevention of UTI in ASB (LoE 2, GoR B)

\section{Bottom line}

Observational data show mixed results regarding the treatment of asymptomatic bacteriuria. Epidemiological studies show that asymptomatic bacteriuria is common in catheter-dependent patients with NB (14-16), and bacterial recurrence after antibiotic treatment is expected in these patients (17-19).

\section{Summary of evidence}

A cohort study of 46 patients with NB secondary to SCI found that treatment of asymptomatic bacteriuria failed to reduce the rate of infection when compared to treatment of complicated infections only (9). Two additional small 
Table 1 Consensus recommendations for UTI prevention in ASB

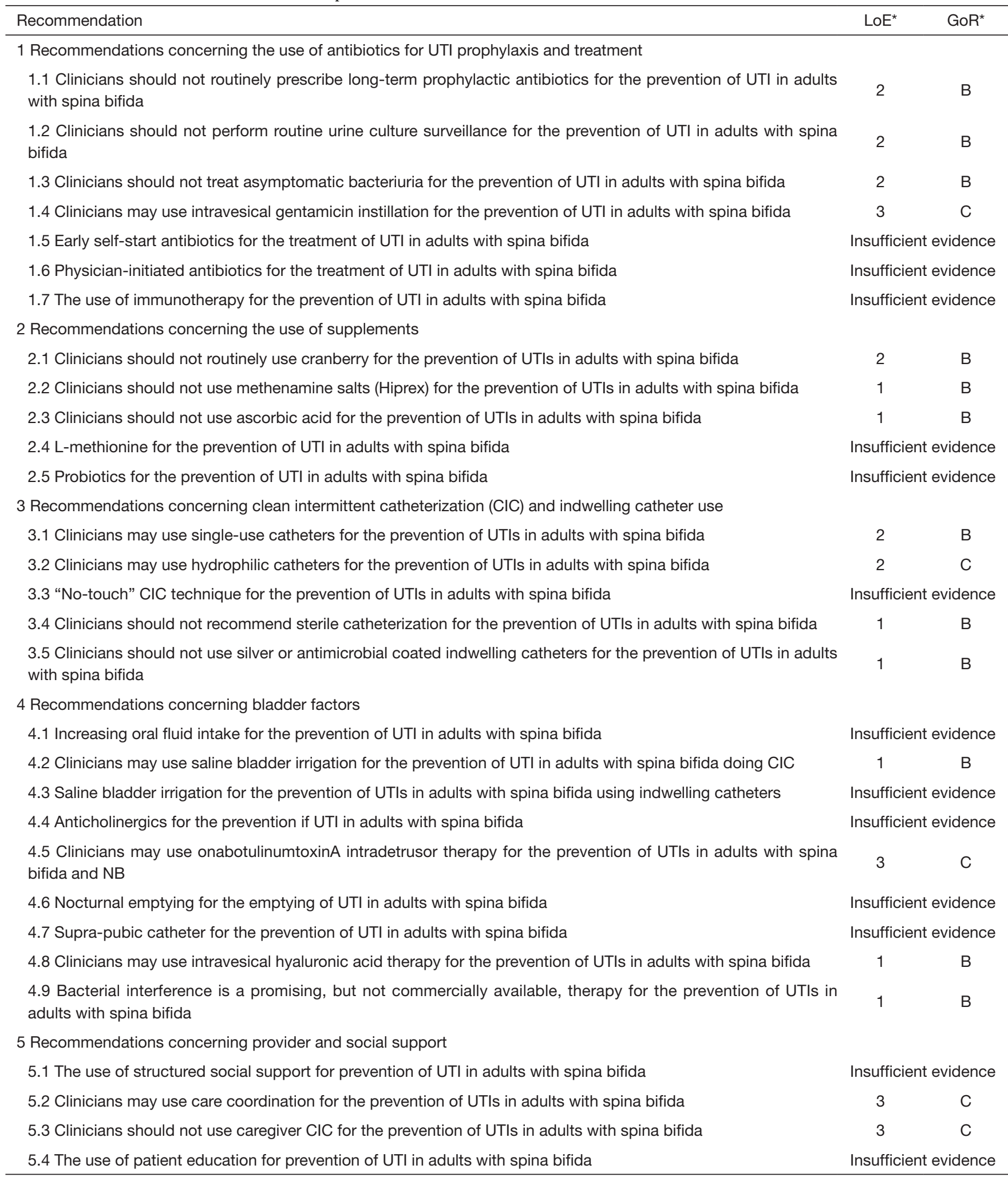

*, scored according to Oxford Level of Evidence (LoE) and Grade of Recommendation (GoR). UTI, urinary tract infection; ASB, adults with spina bifida; NB, neurogenic bladder. 


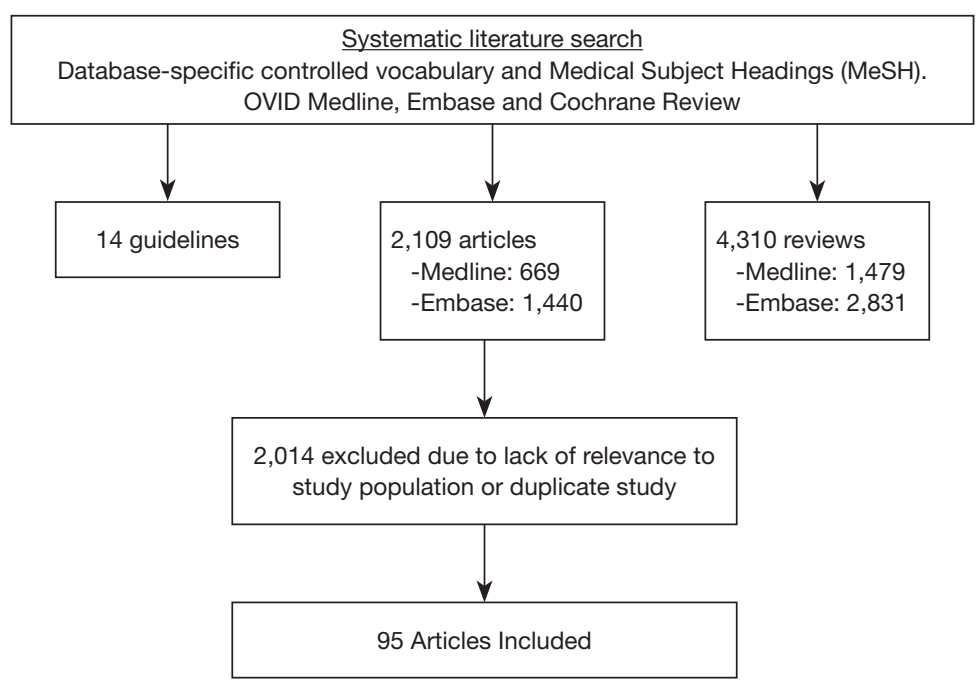

Figure 2 Flow diagram of study identification, eligibility, and inclusion.

observational studies assessed possible sequelae of not treating asymptomatic bacteriuria in patients with $\mathrm{NB}$ and found no increased incidence of upper tract damage or urosepsis $(20,21)$.

Clinicians may use intravesical gentamicin instillation for the prevention of UTI in adults with spina bifida (LoE 3, GoR C)

\section{Bottom line}

Based upon limited observational studies, gentamicin instillation may be an effective option to prevent UTI in ASB.

\section{Summary of evidence}

Antibiotic instillation refers to antibiotic solution being gently infused into the bladder with no mechanical washing. Dilute gentamicin is a common antibiotic utilized, given known effectiveness against uropathogens. No RCT was identified that addressed the use of gentamicin to prevent UTI in NB patients. A single retrospective quasiexperimental study assessing 22 patients with NB before and after the use of intravesical gentamicin found patients used less oral antibiotics after gentamicin instillations were initiated, had fewer symptomatic UTI's and fewer courses of UTI treatment (22). Another retrospective study of 80 children (54 with $\mathrm{NB}$ ) assessed intravesical gentamicin for UTI refractory to two courses of oral antibiotics or had asymptomatic Pseudomonas or Proteus bacteriuria. There were 21 cases of breakthrough UTI during treatment with 5 cases resistant to gentamicin on urine culture (23). A third study including 10 children with bacteriuria doing CIC found that none had a positive urine culture after 7 days of gentamicin instillation (24).

Early self-start antibiotics for the treatment of UTI in adults with spina bifida: insufficient evidence Summary of evidence

Self-start antibiotics is a recognized treatment option for young women with recurrent UTI who can reliably identify their symptoms. This practice proves difficult to adapt to patients with NB owing to a lack of usual symptoms leading to poor UTI diagnostic accuracy. In a prospective study of 147 patients with SCI who presented to the emergency room with symptoms they attributed to a UTI, Linsenmeyer et al. showed that only $61 \%$ accurately predicted a UTI based on the NIDRR consensus definition (25). No studies examining the effectiveness of self-start antibiotics in patients with NB were identified.

\section{Physician-initiated antibiotics for the treatment of} UTI in adults with spina bifida: insufficient evidence, additional research is needed

\section{Bottom line}

Culture-directed antibiotics should be used to treat UTI; however, there is no data specific to empiric antibiotic selection and limited information regarding optimal antibiotic dosages and duration of use in ASB or patients with NB. Empiric antibiotics for ASB is challenging due to resistance patterns.

\section{Summary of evidence}

Multicenter European surveys have highlighted that 
while centers have protocols for management of UTI in $\mathrm{NB}$, they are not standardized and many do not follow guidelines $(26,27)$. A single RCT showed that three days of ciprofloxacin was inferior to 14 days of therapy to clear UTI in patients with SCI (28). A retrospective study assessing antibiotic duration in intervals of $<10,10-15,>15$ days found no difference between groups of SCI patients irrespective of antibiotic choice (29).

The use of immunotherapy for the prevention of UTI in adults with spina bifida: insufficient but promising evidence, further research is recommended

\section{Bottom line}

UTI immunotherapy is defined as the prevention or treatment of UTI with substances that stimulate an immune response; these include vaccines or antigen immunostimulation. There is some evidence for immunotherapy in NB populations and in women with recurrent UTI. These data suggest that immunotherapy may be a promising treatment option for ASB.

\section{Summary of evidence}

A single cross-over RCT found a decrease in bacteriuria and a trend towards UTI reduction in 70 SCI patients treated with oral immunostimulatory fractions extracted from lyophilized E. coli in capsule form (30). A placebo controlled RCT found a significant decrease in UTI rates among women with normal bladder function and recurrent UTI who were treated with vaginal mucosal immunization (31).

\section{Recommendations concerning the use of supplements}

Clinicians should not routinely recommend cranberry for the prevention of UTIs in adults with spina bifida (LOE 2, GoR B)

\section{Bottom line}

Cranberry is a common folk remedy for UTI prevention. Large well-designed RCTs found no UTI benefit in NB populations, while smaller RCTs showed mixed results.

\section{Summary of evidence}

Two double-blind RCTs found no benefit in UTI prevention with the use of cranberry extract in patients with NB secondary to SCI or MS $(32,33)$. Smaller cross-over RCTs showed mixed results: two studies looking at adults with NB secondary to SCI and children with NB on CIC found no reduction in UTI $(34,35)$. Two other cross-over RCTs looking at children with NB secondary to spina bifida and patients with SCI and recurrent UTIs found there was a significant UTI reduction $(36,37)$.
Clinicians should not use methenamine salts (Hiprex) for the prevention of UTIs in adults with spina bifida (LoE 1, GoR B)

\section{Bottom line}

Methenamine salts are used in UTI prevention. It is postulated that the salts break down in acidic $\mathrm{pH}$ within the urine to form bactericidal formaldehyde and ammonia. A large and well-designed RCT found no UTI benefit in patients with SCI.

\section{Summary of evidence}

A single double-blind RCT assessing methenamine salt use for the prevention of UTI in 305 patients with NB secondary to SCI found patients randomized to methenamine salts had no difference in UTI-free period compared to placebo (32).

Clinicians should not use ascorbic acid for the prevention of UTIs in adults with spina bifida (LoE 1, GoR B)

Bottom line

Ascorbic acid is a urine acidifier. No studies were identified that demonstrated a benefit to its use in UTI or bacteriuria prevention.

\section{Summary of evidence}

No studies were identified that addressed the use of ascorbic acid for UTI prevention. A single RCT assessing the use of ascorbic acid for the prevention of bacteriuria was identified. In 161 hospitalized patients with SCI and a history of UTI who were randomized to no prophylactic agent (control), ascorbic acid, or antibiotics, there was no reduction in bacteriuria with ascorbic acid use (38).

L-methionine for the prevention of UTI in adults with spina bifida: insufficient evidence

\section{Bottom line}

L-methionine is another urine acidifier. No studies assessing the use of L-methionine for the prevention of UTI were identified.

Summary of evidence

Not applicable.

Probiotics for the prevention of UTI in adults with spina bifida: insufficient evidence

\section{Bottom line}

Probiotics are proposed to improve the microbiome of patients to prevent UTIs. There are no published studies evaluation their use in UTI prevention in people with NB and the evidence is mixed in other populations. 


\section{Summary of evidence}

The only study of probiotics for UTI prevention in NB is currently ongoing; only the protocol has been published (39). Three RCTs assessing the use of probiotics to prevent UTI in women with normal bladder function found mixed results, with one trial having positive results and two that did not show a difference between probiotic and control groups (40-42).

\section{Recommendations concerning IC and indwelling catheter use}

Clinicians may use single-use catheters for the prevention of UTIs in adults with spina bifida (LoE 2, GoR B)

\section{Bottom line}

The role of single-use catheters $v s$. reusable catheters in $\mathrm{CIC}$ is debated. This debate has largely played out through a recent meta-analysis that reviewed a 2014 Cochrane review's data $(43,44)$. The original 2014 review found no difference in UTI rates between the two methods and recommended reusable catheters. The 2017 review found many issues with the data handling and on reanalysis of the same data found a trend towards benefit to single-use catheters. This study has led to the redaction of the 2014 review. In alignment with this recent thorough analysis, single-use catheters may be recommended for UTI prevention in adults with spina bifida.

\section{Summary of the evidence}

A 16-week crossover RCT of single-use versus reusable non-coated catheters in 23 patients doing twice daily CIC showed no difference in the rate of UTI, bacteriuria, or cystitis symptoms (45). Another trial of ten children using CIC to manage NB found there was no significant difference in the rate of bacteriuria between single-use and reusable catheters (46). The 2017 review mentioned above performed an analysis demonstrating that there was a trend, albeit small, towards the benefit of single-use catheters for the prevention of UTIs (43).

\section{Clinicians may use hydrophilic catheters for the} prevention of UTIs in adults with spina bifida (LoE 2, GoR C)

\section{Bottom line}

The use of hydrophilic catheters is controversial. Four of seven RCTs support their use for UTI prevention. The two largest RCTs were both positive trials. Of note, these studies were industry-funded and had $<80 \%$ follow-up. Large observational studies have found no UTI benefit. Hydrophilic catheters may be recommended for UTI prevention in adults with spina bifida.

\section{Summary of evidence}

Seven RCTs were identified that assessed UTI frequency in NB patients using hydrophilic CIC. The two largest studies had poor study completion rates and included 114 and 57 patients who were followed over 6 and 12 months, respectively. Both studies found significant reductions in UTI $(47,48)$. Of the smaller 5 RCTs: 2 found a UTI reduction (49,50), 2 found no UTI benefit with hydrophilic catheter use, and 1 found increased UTI associated with hydrophilic catheters (51-53). Two survey-based observational studies, including one national Canadian survey with 912 responders, reported no benefit with hydrophilic catheter use $(54,55)$.

The use of a "no-touch" IC technique for the prevention of UTIs in adults with spina bifida: insufficient evidence

\section{Bottom line}

No-touch IC is a technique specifically designed for catheter insertion without the user touching the catheter. Catheterization is achieved via an insertion tip, protective sleeve/grip, or a closed system. No studies were identified to assess the utility of no-touch IC to prevent UTI in NB.

\section{Summary of evidence}

Not applicable.

\section{Clinicians should not recommend sterile} catheterization for the prevention of UTIs in adults with spina bifida (LoE 1, GoR B)

\section{Bottom line}

Since Dr. Lapides' 1972 report (56), CIC has consistently been shown to be a cost-effective and preferred alternative to sterile IC. Primary data shows no difference in UTI risk between CIC and sterile catheterization.

\section{Summary of evidence}

Three prospective studies, including two RCTs, were identified assessing CIC $v s$. sterile catheterization techniques in patients with NB. These studies involved 29-46 patients and followed them for 1-3 months. None of the three studies found any difference in UTI rates between groups (57-59). 
Clinicians should not use silver or antimicrobial coated indwelling catheters for the prevention of UTIs in adults with spina bifida (LoE 1, GoR B)

\section{Bottom line}

IC is generally the preferred bladder management technique for patients with NB. In patients who cannot do IC, indwelling catheters is an alternative. No primary studies specific to NB populations were identified. Large studies of all-comer hospitalized patients showed mixed results with respect to CA-UTI rates when randomized to the use of silver or antimicrobial coated catheters.

\section{Summary of evidence}

Three large studies (2 RCT, 1 cohort) assessed coated indwelling catheters used in all-comer hospitalized patients and showed conflicting evidence: The largest study, involving 27,000 patients found a significant UTI reduction (60). The other two smaller but very large studies found no difference in UTI rates with silver (cohort study covering $>56,000$ patient days) or anti-bacterial (7,102 patients) coated catheters $(61,62)$.

\section{Recommendations concerning bladder factors}

Increasing fluid intake for the prevention of UTI in adults with spina bifida: insufficient evidence

\section{Bottom line}

Some practitioners suggest that increased fluid intake may decrease bacterial colony counts through dilution or increased bladder cycled secondary to increased fluids. However, no studies assessing fluid intake relevant to NB patients were identified.

\section{Summary of data}

Not applicable.

Clinicians may use saline bladder irrigation for the prevention of UTI in adults with spina bifida doing CIC (LoE 1, GoR B)

Bottom line

An RCT involving only ASB patients doing CIC with 10year follow-up demonstrated a significant reduction in UTI with daily saline bladder irrigation.

\section{Summary of evidence}

Seventy-five total patients with ASB were randomized to daily saline irrigation with volumes of 60,120 or $240 \mathrm{~mL}$ of saline daily and followed for 10 years. The primary outcome was bladder calculi recurrence with a secondary outcome of total UTI. Results showed the group doing high volume $(240 \mathrm{~mL})$ irrigations has significantly fewer total UTI than the mid $(120 \mathrm{~mL})$ and small volume $(60 \mathrm{~mL})$ irrigation groups (63).

The use of saline bladder irrigation for the prevention of UTI in adults with spina bifida using indwelling catheters: insufficient evidence

\section{Bottom line}

Indwelling catheters are associated with higher rates of bacteriuria and UTI than IC. Bladder irrigation is aimed to flush out these bacteria.

Summary of evidence

A single 2006 RCT assessed bacteriuria rates in patients with NB managed by indwelling catheter who were randomized to irrigate their bladders twice daily for 8 weeks with $30 \mathrm{~mL}$ of sterile saline, acetic acid, or neomycinpolymyxin solution. The study found none of the three irrigates had a difference on the degree of bacteriuria or pyuria (64).

The use of anticholinergics for the prevention if UTI in adults with spina bifida: insufficient evidence Bottom line

There is strong laboratory evidence that increased bladder pressures are associated with bacterial translocation across the urothelium (65). Furthermore, there is evidence in people with NB that increased bladder pressures are associated with more frequent and more severe UTIs (66). Therefore, the lack of literature assessing the relationship between oral anticholinergics and UTI is surprising, we hypothesize that the pervasiveness of anticholinergics in NB patients has prevented rigorous analysis of any association that may exist. Despite the lack of evidence specific to oral anticholinergics, there is a broad and growing body of evidence that intravesical pressure optimization may prevent UTI.

Summary of evidence

No studies specifically examined the incidence of UTIs with $v s$. without oral anticholinergics.

Clinicians may use onabotulinumtoxinA intradetrusor therapy for the prevention of UTIs in adults with spina bifida and NB (LoE 3, GoR C)

\section{Bottom line}

There is data from well-designed observational studies suggesting improved detrusor pressure can decrease UTI. OnabotulinumtoxinA injections may be a valid therapeutic option to reduce UTI in adults with spina bifida.

\section{Summary of evidence}

Two quasi-experimental studies (41 and 30 patients) and one 
retrospective cohort (214 patients) assessing long-term UTI rates in NB patients before and after onabotulinumtoxinA injection both found a significant UTI reduction in the interval after onabotulinumtoxinA injections (67-69). A small case series of seven children with NDO saw a UTI reduction but not a pyelonephritis reduction in two children who were prone to upper tract infections (70).

\section{The use of nocturnal emptying for UTI prevention in adults with spina bifida: insufficient evidence \\ Bottom line}

Nocturnal emptying involves allowing the bladder to continuous drain via catheter overnight then resuming IC during the day. It is thought to reduce UTI risk by keeping the bladder empty and decompressed while the patient is presumably sleeping and would not be performing IC. The limited available evidence is promising.

\section{Summary of evidence}

Two observational studies in NB patients and one pediatric study, all without controls, report UTI reduction with nocturnal emptying. Two additional studies were small case series in children; both reported fewer UTIs after initiation of nocturnal emptying but were limited by small study sizes $(71,72)$. A study of children with polyuria demonstrated decrease in mean hospitalizations for febrile UTI from 1.7 to 0.4 with nocturnal bladder emptying (73).

The use of a suprapubic tube (SPT) for the prevention of UTI in adults with spina bifida: insufficient evidence Bottom line

SPT is commonly believed by patients and physicians to have a lower risk of UTI compared to indwelling urethral catheter. There is insufficient data to support or refute this notion.

\section{Summary of evidence}

There is insufficient evidence to compare UTI risk associated with SPT $v s$. indwelling urethral catheter or CIC. However, SPT is preferable to indwelling urethral catheter for other reasons; namely, because of the urethral complications that can occur with long-term indwelling urethral catheter (74). A single observational study of 61 quadriplegics followed an average of 9 years found no significant difference in UTI incidence between CIC and SPT (75). Two retrospective cohort studies found conflicting results when comparing UTI rates between SPT and indwelling urethral catheter. The first, a study following 125 Nigerian patients with NB found a significant significantly lower incidence of septicemia with SPT than with indwelling urethral catheter (76). The second, a U.S. study of 44 NB patients found no difference in UTI rates between indwelling urethral catheter and SPT (77).

Clinicians may use intravesical hyaluronic acid (HA) therapy for the prevention of UTIs in adults with spina bifida (LoE 1, GoR B)

\section{Bottom line}

HA is a constituent of the bladder surface glycosaminoglycan layer which is a barrier to the urothelium and may help to prevent UTIs. All studies identified support the use of intravesical HA for UTI prevention. Intravesical HA may be a valid therapeutic option to reduce UTI in ASB.

\section{Summary of evidence}

A 2-year case series of eight children with $\mathrm{NB}$ or vesicoureteral reflux receiving weekly HA treatment for 1 month, with concurrent TMP-SMX therapy, showed complete (no UTI) and partial response rates (>50\% UTI reduction) in $62.5 \%$ and $25 \%$ of patients, respectively (78). Another case series of 10 patients with NB and recurrent UTI who received $40 \mathrm{mg}$ HA weekly for the first month, then monthly for 3 months found a significant reduction in recurrent UTI after the 4-month treatment period (79).

A double-blind, RCT of 57 neurologically intact women showed a reduction in UTI and a significant increase in time to UTI recurrence with intravesical HA (80). These results are supported by another RCT (81), quasi-experimental studies $(79,82)$ and a retrospective cohort study $(83)$ that all showed positive results.

\section{Bacterial interference is a promising, but not} commercially available, therapy for the prevention of UTIs in adults with spina bifida. Further research is needed (LoE 1, GoR B)

\section{Bottom line}

Bacterial interference is the limitation of growth of pathogenic bacteria via the deliberate colonization with benign microorganisms. Small RCTs consistently have shown a significant reduction in UTI rates in patients with NB after inoculation with non-pathogenic E. coli strains. The clinical utility of bacterial interference is currently limited because of low inoculation rates and no commercially available therapies.

\section{Summary of evidence}

Three prospective blinded RCTs were identified assessing the effects of bacterial interference on UTI rates in patients with NB. These three studies followed a total of 74 patients (study size was limited because colonization rates were low) 
inoculated with non-pathogenic E. coli over $>12$ months. All three studies found a significant reduction in UTI rates compared to saline injection controls (84-86). Three quasiexperimental studies including 65 patients with NB also demonstrate a reduction in UTI frequency post-inoculation (87-89).

\section{Recommendations concerning provider and social support}

The use of structured social support for prevention of UTI in adults with spina bifida: insufficient evidence Summary of evidence

Structured social support was defined as any established system to check-in or provide support outside a traditional clinic setting. Two studies assessed the effects of telemedicine on UTI in NB with conflicting results: a 3-month study following 26 patients showed no UTI reduction after a telemedicine intervention (90). A study assessing 49 patients with $\mathrm{NB}$ found a significant reduction in UTI with telemedicine (91).

Clinicians may use care coordination for the prevention of UTIs in adults with spina bifida (LoE 3, GoR C)

Bottom line

Care coordination is the act of organizing delivery of health care services with the goal to improve outcomes. This can be in the form of a dedicated nurse who interacts with patients frequently and helps coordinate their care delivery across several providers. No literature identified assessed the impact of care coordination on UTI rates in any NB population. An expanded search of care coordination on other health outcomes in ASB was conducted. Further research is needed on the effect of care coordination and UTI rates in ASB.

\section{Summary of evidence}

Dicianno et al. reported on 65 adults with SB or SCI. The two-year intervention was a quarterly at home visit by a registered nurse. Visits targeted prevention in the following domains: decubitus ulcer, depression, bladder, bowel, nutrition and physical activity. Goal-setting and education were integral. At the end of the intervention, patients showed an improvement in all categories, and there was a small net financial savings from the nurse coordinator (92). Similarly, a case series of 15 adolescents with spina bifida who received a nurse-led virtual clinic via Skype reported increased confidence after the intervention (93).

Three additional publications report on the methods and qualitative success, without any objective measures, of care coordination interventions. Common components of the interventions are: early health-care needs assessment, interprovider communication, addressing family concerns, and follow-up (94-96).

\section{Clinicians should not use caregiver CIC for the prevention of UTIs in adults with spina bifida (LoE 3, GoR C)}

Bottom line

A limited body of data across the primary and secondary literature has consistently found or concluded there is no benefit in UTI risk with caregivers performing the CIC, rather than the patient.

\section{Summary of evidence}

A single study assessing self CIC $v s$. catheter team CIC in 73 patients hospitalized acutely after SCI found no significant difference regarding the rate of urinary infection or the incidence of urethral trauma (97).

The use of patient education for prevention of UTI in adults with spina bifida: insufficient evidence Summary of evidence

Although patient education is an important component of proper bladder management in NB, we could not identify any studies that linked patient education to UTI rates. A single RCT of 56 patients with NB and recurrent UTI were randomized to a UTI reduction education program that included: written material on UTIs, a self-administered test, a review by nurse and physician, and a follow-up telephone call $v s$. no intervention over a 6-month period. The study results trended toward a UTI reduction but was not statistically significant (98).

\section{Conclusions}

There are few studies assessing UTI prevention specifically for ASB. These recommendations, which are largely based on data from populations similar to ASB, may help to guide clinicians. The following interventions have not been shown to be beneficial in UTI prevention and should not be used routinely: cranberry, methenamine salts, prophylactic antibiotics, surveillance cultures, treating asymptomatic bacteriuria, and sterile or caregiver IC. Beneficial interventions based on the available evidence were saline bladder irrigation, provider care coordination, and a new catheter with each catheterization. Furthermore, many emerging therapies demonstrated positive results including: intradetrusor onabotulinumtoxinA, hydrophilic 
IC, intravesical gentamicin and HA. Areas of insufficient evidence where future research could be particularly impactful are physician-initiated empiric antibiotic regimens and definitive antibiotic duration of use, oral anticholinergics, bacterial interference, and social support/ patient education measures.

The scope of the 30 outcomes questions covered is intended to be relevant to any provider making decisions regarding bladder management in ASB. To our knowledge, a comprehensive review within this patient population has not been previously reported. Because we found little primary literature on UTI prevention and management in ASB, we expanded the scope of our search to include UTI prevention and management strategies in other relevant populations; it was challenging to ensure that the expanded search was conducted systematically without yielding an over-abundance of references. Generally, any literature identified from a NB population was included; when there were fewer than five articles identified for a specific outcome question, the review was expanded to include the highest quality evidence in neurologically intact patients.

The only RCT identified in the entire review specific to UTI prevention in ASB was the high-quality 2016 study by Husmann reporting a significant UTI reduction over 2 years of high-volume saline irrigation compared to lower volume irrigations. The use of new or single-use catheters is a highly debated topic. Our positive recommendation aligns with the recent analysis by Christison et al. (43).

Spina bifida is no longer an exclusively pediatric disease, and urologic congenitalism is growing field in need for further research (99). Adults with spina bifida, like many other high-risk populations, often struggle with the morbidity of UTIs. There is a stark paucity of evidence specific to the ASB population. This review and practice policy recommendations are intended to be a guide for UTI prevention in this population and to serve to highlight opportunities for much needed future research efforts.

\section{Acknowledgements}

The authors would like to thank Philipp Dahm, MD; Jairam R. Eswara, MD; Michael J. Kennelly, MD, FACS, FPMRS; Rose Khavari, MD; Sara Lenherr, MD, MS; Jeremy B. Myers, MD, FACS; Yahir Santiago-Lastra, MD; John T. Stoffel, MD; Blayne Welk, MD, FRCSC, MSC.

\section{Footnote}

Conflicts of Interest: The authors have no conflicts of interest to declare.

\section{References}

1. Wang HH, Wiener JS, Ross SS, et al. Emergent care patterns in patients with spina bifida: a case-control study. J Urol 2015;193:268-73.

2. Thorup J, Biering-Sorensen F, Cortes D. Urological outcome after myelomeningocele: 20 years of follow-up. BJU Int 2011;107:994-9.

3. The prevention and management of urinary tract infections among people with spinal cord injuries. National Institute on Disability and Rehabilitation Research Consensus Statement. January 27-29, 1992. J Am Paraplegia Soc 1992;15:194-204.

4. Gribble MJ, Puterman ML. Prophylaxis of urinary tract infection in persons with recent spinal cord injury: a prospective, randomized, double-blind, placebo-controlled study of trimethoprim-sulfamethoxazole. Am J Med 1993;95:141-52.

5. Johnson HW, Anderson JD, Chambers GK, et al. A shortterm study of nitrofurantoin prophylaxis in children managed with clean intermittent catheterization. Pediatrics 1994;93:752-5.

6. Schlager TA, Anderson S, Trudell J, et al. Nitrofurantoin prophylaxis for bacteriuria and urinary tract infection in children with neurogenic bladder on intermittent catheterization. J Pediatr 1998;132:704-8.

7. Biering-Sørensen F, Høiby N, Nordenbo A, et al. Ciprofloxacin as prophylaxis for urinary tract infection: prospective, randomized, cross-over, placebo controlled study in patients with spinal cord lesion. J Urol 1994;151:105-8.

8. Sandock DS, Gothe BG, Bodner DR. Trimethoprimsulfamethoxazole prophylaxis against urinary tract infection in the chronic spinal cord injury patient. Paraplegia 1995;33:156-60.

9. Mohler JL, Cowen DL, Flanigan RC. Suppression and treatment of urinary tract infection in patients with an intermittently catheterized neurogenic bladder. J Urol 1987;138:336-40.

10. Clarke SA, Samuel M, Boddy SA. Are prophylactic antibiotics necessary with clean intermittent catheterization? A randomized controlled trial. J Pediatr 
Surg 2005;40:568-71.

11. Salomon J, Denys P, Merle C, et al. Prevention of urinary tract infection in spinal cord-injured patients: safety and efficacy of a weekly oral cyclic antibiotic (WOCA) programme with a 2 year follow-up-an observational prospective study. J Antimicrob Chemother 2006;57:784-8.

12. Poirier C, Dinh A, Salomon J, et al. Prevention of urinary tract infections by antibiotic cycling in spinal cord injury patients and low emergence of multidrug resistant bacteria. Med Mal Infect 2016;46:294-9.

13. Salomon J, Schnitzler A, Ville Y, et al. Prevention of urinary tract infection in six spinal cord-injured pregnant women who gave birth to seven children under a weekly oral cyclic antibiotic program. Int J Infect Dis 2009;13:399-402.

14. Schlager TA, Dilks S, Trudell J, et al. Bacteriuria in children with neurogenic bladder treated with intermittent catheterization: natural history. J Pediatr 1995;126:490-6.

15. Dilks SA, Schlager T, Kopco JA, et al. Frequency and correlates of bacteriuria among children with neurogenic bladder. South Med J 1993;86:1372-5.

16. Svensson E, Ertzgaard P, Forsum U. Bacteriuria in spinal cord injured patients with neurogenic bladder dysfunction. Ups J Med Sci 2004;109:25-32.

17. Waites KB, Canupp KC, DeVivo MJ. Eradication of urinary tract infection following spinal cord injury. Paraplegia 1993;31:645-52.

18. Donovan WH, Hull R, Rossi CD. Analysis of gram negative recolonization of the neuropathic bladder among patients with spinal cord injuries. Spinal Cord 1996;34:587-91.

19. Penders J, Huylenbroeck AA, Everaert K, et al. Urinary infections in patients with spinal cord injury. Spinal Cord 2003;41:549-52.

20. Paradella AC, Musegante AF, Brites C. Comparison of different antibiotic protocols for asymptomatic bacteriuria in patients with neurogenic bladder treated with botulinum toxin A. Braz J Infect Dis 2016;20:623-6.

21. Ottolini MC, Shaer CM, Rushton HG, et al. Relationship of asymptomatic bacteriuria and renal scarring in children with neuropathic bladders who are practicing clean intermittent catheterization. J Pediatr 1995;127:368-72.

22. Cox L, He C, Bevins J, et al. Gentamicin bladder instillations decrease symptomatic urinary tract infections in neurogenic bladder patients on intermittent catheterization. Can Urol Assoc J 2017;11:E350-4.

23. Defoor W, Ferguson D, Mashni S, et al. Safety of gentamicin bladder irrigations in complex urological cases. J Urol 2006;175:1861-4.

24. Wan J, Kozminski M, Wang SC, et al. Intravesical instillation of gentamicin sulfate: in vitro, rat, canine, and human studies. Urology 1994;43:531-6.

25. Linsenmeyer TA, Oakley A. Accuracy of individuals with spinal cord injury at predicting urinary tract infections based on their symptoms. J Spinal Cord Med 2003;26:352-7.

26. Zegers BSH Winkler-Seinstra PL, Uiterwaal CS, et al. Urinary tract infections in children with spina bifida: an inventory of 41 European centers. Pediatr Nephrol 2009;24:783-8.

27. Pannek J. Treatment of urinary tract infection in persons with spinal cord injury: guidelines, evidence, and clinical practice. A questionnaire-based survey and review of the literature. J Spinal Cord Med 2011;34:11-5.

28. Dow G, Rao P, Harding G, et al. A prospective, randomized trial of 3 or 14 days of ciprofloxacin treatment for acute urinary tract infection in patients with spinal cord injury. Clin Infect Dis 2004;39:658-64.

29. Dinh A, Toumi A, Blanc C, et al. Management of febrile urinary tract infection among spinal cord injured patients. BMC Infect Dis 2016;16:156.

30. Hachen HJ. Oral immunotherapy in paraplegic patients with chronic urinary tract infections: a double-blind, placebo-controlled trial. J Urol 1990;143:759-62; discussion 762-3.

31. Hopkins WJ, Elkahwaji J, Beierle LM, et al. Vaginal mucosal vaccine for recurrent urinary tract infections in women: results of a phase 2 clinical trial. J Urol 2007;177:1349-53; quiz 1591.

32. Lee BB, Haran MJ, Hunt LM, et al. Spinal-injured neuropathic bladder antisepsis (SINBA) trial. Spinal Cord 2007;45:542-50.

33. Gallien P, Amarenco G, Benoit N, et al. Cranberry versus placebo in the prevention of urinary infections in multiple sclerosis: a multicenter, randomized, placebo-controlled, double-blind trial. Mult Scler 2014;20:1252-9.

34. Linsenmeyer TA, Harrison B, Oakley A, et al. Evaluation of cranberry supplement for reduction of urinary tract infections in individuals with neurogenic bladders secondary to spinal cord injury. A prospective, doubleblinded, placebo-controlled, crossover study. J Spinal Cord Med 2004;27:29-34.

35. Schlager TA, Anderson S, Trudell J, et al. Effect of cranberry juice on bacteriuria in children with neurogenic bladder receiving intermittent catheterization. J Pediatr 
1999;135:698-702.

36. Mutlu H, Ekinci Z. Urinary tract infection prophylaxis in children with neurogenic bladder with cranberry capsules: randomized controlled trial. ISRN Pediatr 2012;2012:317280.

37. Hess MJ, Hess PE, Sullivan MR, et al. Evaluation of cranberry tablets for the prevention of urinary tract infections in spinal cord injured patients with neurogenic bladder. Spinal Cord 2008;46:622-6.

38. Kuhlemeier KV, Stover SL, Lloyd LK. Prophylactic antibacterial therapy for preventing urinary tract infections in spinal cord injury patients. J Urol 1985;134:514-7.

39. Lee BB, Toh SL, Ryan S, et al. Probiotics (LGG$\mathrm{BB} 12$ or RC14-GR1) versus placebo as prophylaxis for urinary tract infection in persons with spinal cord injury (ProSCIUTTU): a study protocol for a randomised controlled trial. BMC Urol 2016;16:18.

40. Beerepoot MA, ter Riet G, Nys S, et al. Lactobacilli vs antibiotics to prevent urinary tract infections: a randomized, double-blind, noninferiority trial in postmenopausal women. Arch Intern Med 2012;172:704-12.

41. Kontiokari T, Sundqvist K, Nuutinen M, et al. Randomised trial of cranberry-lingonberry juice and Lactobacillus GG drink for the prevention of urinary tract infections in women. BMJ 2001;322:1571.

42. Stapleton AE, Au-Yeung M, Hooton TM, et al. Randomized, placebo-controlled phase 2 trial of a Lactobacillus crispatus probiotic given intravaginally for prevention of recurrent urinary tract infection. Clin Infect Dis 2011;52:1212-7.

43. Christison K, Walter M, Wyndaele JJM, et al. Intermittent Catheterization: The Devil Is in the Details. J Neurotrauma 2018. [Epub ahead of print].

44. Prieto J, Murphy CL, Moore KN, et al. WITHDRAWN: Intermittent catheterisation for long-term bladder management. Cochrane Database Syst Rev 2017;8:CD006008.

45. Leek H, Stephenson Z, Reus A, et al. Clean intermittent self-catheterisation: A randomised controlled crossover trial of single-use versus multiple re-use of non-coated catheters; is cystitis rate altered? Neurourol Urodyn 2013;32:759-60.

46. Schlager TA, Clark M, Anderson S. Effect of a singleuse sterile catheter for each void on the frequency of bacteriuria in children with neurogenic bladder on intermittent catheterization for bladder emptying. Pediatrics 2001;108:E71.
47. Cardenas DD, Moore KN, Dannels-McClure A, et al. Intermittent catheterization with a hydrophilic-coated catheter delays urinary tract infections in acute spinal cord injury: a prospective, randomized, multicenter trial. PM R 2011;3:408-17.

48. De Ridder DJ, Everaert K, Fernández LG, et al. Intermittent catheterisation with hydrophilic-coated catheters (SpeediCath) reduces the risk of clinical urinary tract infection in spinal cord injured patients: a prospective randomised parallel comparative trial. Eur Urol 2005;48:991-5.

49. Vapnek JM, Maynard FM, Kim J. A prospective randomized trial of the LoFric hydrophilic coated catheter versus conventional plastic catheter for clean intermittent catheterization. J Urol 2003;169:994-8.

50. DeFoor W, Reddy P, Reed M, et al. Results of a prospective randomized control trial comparing hydrophilic to uncoated catheters in children with neurogenic bladder. J Pediatr Urol 2017;13:373.e1-373.e5.

51. Cardenas DD, Hoffman JM. Hydrophilic catheters versus noncoated catheters for reducing the incidence of urinary tract infections: a randomized controlled trial. Arch Phys Med Rehabil 2009;90:1668-71.

52. Kiddoo D, Sawatzky B, Bascu CD, et al. Randomized Crossover Trial of Single Use Hydrophilic Coated vs Multiple Use Polyvinylchloride Catheters for Intermittent Catheterization to Determine Incidence of Urinary Infection. J Urol 2015;194:174-9.

53. Sarica S, Akkoc Y, Karapolat H, et al. Comparison of the use of conventional, hydrophilic and gel-lubricated catheters with regard to urethral micro trauma, urinary system infection, and patient satisfaction in patients with spinal cord injury: a randomized controlled study. Eur J Phys Rehabil Med 2010;46:473-9.

54. Spinu A, Onose G, Daia C, et al. Intermittent catheterization in the management of post spinal cord injury (SCI) neurogenic bladder using new hydrophilic, with lubrication in close circuit devices--our own preliminary results. J Med Life 2012;5:21-8.

55. Woodbury MG, Hayes KC, Askes HK. Intermittent catheterization practices following spinal cord injury: a national survey. Can J Urol 2008;15:4065-71.

56. Lapides J, Diokno AC, Silber SJ, et al. Clean, intermittent self-catheterization in the treatment of urinary tract disease. J Urol 1972;107:458-61.

57. King RB, Carlson CE, Mervine J, et al. Clean and sterile intermittent catheterization methods in hospitalized patients with spinal cord injury. Arch Phys Med Rehabil 
1992;73:798-802.

58. Moore KN, Burt J, Voaklander DC. Intermittent catheterization in the rehabilitation setting: a comparison of clean and sterile technique. Clin Rehabil 2006;20:461-8.

59. Prieto-Fingerhut T, Banovac K, Lynne CM. A study comparing sterile and nonsterile urethral catheterization in patients with spinal cord injury. Rehabil Nurs 1997;22:299-302.

60. Karchmer TB, Giannetta ET, Muto CA, et al. A randomized crossover study of silver-coated urinary catheters in hospitalized patients. Arch Intern Med 2000;160:3294-8.

61. Lai KK, Fontecchio SA. Use of silver-hydrogel urinary catheters on the incidence of catheter-associated urinary tract infections in hospitalized patients. Am J Infect Control 2002;30:221-5.

62. Pickard R, Lam T, Maclennan G, et al. Types of urethral catheter for reducing symptomatic urinary tract infections in hospitalised adults requiring short-term catheterisation: multicentre randomised controlled trial and economic evaluation of antimicrobial- and antiseptic-impregnated urethral catheters (the CATHETER trial). Health Technol Assess 2012;16:1-197.

63. Husmann DA. Long-term complications following bladder augmentations in patients with spina bifida: bladder calculi, perforation of the augmented bladder and upper tract deterioration. Transl Androl Urol 2016;5:3-11.

64. Waites KB, Canupp KC, Roper JF, et al. Evaluation of 3 methods of bladder irrigation to treat bacteriuria in persons with neurogenic bladder. J Spinal Cord Med 2006;29:217-26.

65. Wu J, Miao Y, Abraham SN. The multiple antibacterial activities of the bladder epithelium. Ann Transl Med 2017;5:35.

66. Seki N, Masuda K, Kinukawa N, et al. Risk factors for febrile urinary tract infection in children with myelodysplasia treated by clean intermittent catheterization. Int J Urol 2004;11:973-7.

67. Wefer B, Ehlken B, Bremer J, et al. Treatment outcomes and resource use of patients with neurogenic detrusor overactivity receiving botulinum toxin A (BOTOX) therapy in Germany. World J Urol 2010;28:385-90.

68. Gamé X, Castel-Lacanal E, Bentaleb Y, et al. Botulinum toxin A detrusor injections in patients with neurogenic detrusor overactivity significantly decrease the incidence of symptomatic urinary tract infections. Eur Urol 2008;53:613-8.

69. Jia C, Liao LM, Chen G, et al. Detrusor botulinum toxin
A injection significantly decreased urinary tract infection in patients with traumatic spinal cord injury. Spinal Cord 2013;51:487-90.

70. Do Ngoc Thanh C, Audry G, Forin V. Botulinum toxin type A for neurogenic detrusor overactivity due to spinal cord lesions in children: a retrospective study of seven cases. J Pediatr Urol 2009;5:430-6.

71. Koff SA, Gigax MR, Jayanthi VR. Nocturnal bladder emptying: a simple technique for reversing urinary tract deterioration in children with neurogenic bladder. J Urol 2005;174:1629-31; discussion 1632.

72. Nguyen MT, Pavlock CL, Zderic SA, et al. Overnight catheter drainage in children with poorly compliant bladders improves post-obstructive diuresis and urinary incontinence. J Urol 2005;174:1633-6; discussion 1636.

73. Montané B, Abitbol C, Seeherunvong W, et al. Benecial effects of continuous overnight catheter drainage in children with polyuric renal failure. BJU Int 2003; 92:447-51.

74. Weld KJ, Dmochowski RR. Effect of bladder management on urological complications in spinal cord injured patients. J Urol 2000;163:768-72.

75. Mitsui T, Minami K, Furuno T, et al. Is suprapubic cystostomy an optimal urinary management in high quadriplegics?. A comparative study of suprapubic cystostomy and clean intermittent catheterization. Eur Urol 2000;38:434-8.

76. Nwadiaro HC, Nnamonu MI, Ramyil VM, et al. Comparative analysis of urethral catheterization versus suprapubic cystostomy in management of neurogenic bladder in spinal injured patients. Niger J Med 2007;16:318-21.

77. Vainrib M, Ginsberg DA. Long-term management of neuropathic patients with indwelling transurethral catheter or suprapubic cystostomy: what is better? Neurourol Urodyn 2012;31:1094.

78. Fidan K, Büyükkaragöz B, Özen O, et al. The use of intravesical hyaluronic acid for recurrent urinary tract infections in children: a case-series study. Ren Fail 2015;37:354-8.

79. Alpay H, Yildiz N, Deniz NC, et al. Intravesical hyaluronic acid therapy in recurrent urinary tract infection in patients with spina bifida and neurogenic bladder. Pediatric Nephrology 2014;29:1794.

80. Damiano R, Quarto G, Bava I, et al. Prevention of recurrent urinary tract infections by intravesical administration of hyaluronic acid and chondroitin sulphate: a placebo-controlled randomised trial. Eur Urol 
2011;59:645-51.

81. De Vita D, Giordano S. Effectiveness of intravesical hyaluronic acid/chondroitin sulfate in recurrent bacterial cystitis: a randomized study. Int Urogynecol J 2012;23:1707-13.

82. Sharifiaghdas F, Hamzehi Esfahani N. Intravesical instillation of hyaluranic acid in the treatment of recurrent urinary tract infection in women. Eur Urol 2010;9:616.

83. Constantinides C, Manousakas T, Nikolopoulos P, et al. Prevention of recurrent bacterial cystitis by intravesical administration of hyaluronic acid: a pilot study. BJU Int 2004;93:1262-6.

84. Darouiche RO, Green BG, Donovan WH, et al. Multicenter randomized controlled trial of bacterial interference for prevention of urinary tract infection in patients with neurogenic bladder. Urology 2011;78:341-6.

85. Darouiche RO, Thornby JI, Cerra-Stewart C, et al. Bacterial interference for prevention of urinary tract infection: a prospective, randomized, placebo-controlled, double-blind pilot trial. Clin Infect Dis 2005;41:1531-4.

86. Sundén F, Håkansson L, Ljunggren E, et al. Escherichia coli 83972 bacteriuria protects against recurrent lower urinary tract infections in patients with incomplete bladder emptying. J Urol 2010;184:179-85.

87. Darouiche RO, Donovan WH, Del Terzo M, et al. Pilot trial of bacterial interference for preventing urinary tract infection. Urology 2001;58:339-44.

88. Hull R, Rudy D, Donovan W, et al. Urinary tract infection prophylaxis using Escherichia coli 83972 in spinal cord injured patients. J Urol 2000;163:872-7.

89. Prasad A, Cevallos ME, Riosa S, et al. A bacterial interference strategy for prevention of UTI in persons practicing intermittent catheterization. Spinal Cord 2009;47:565-9.

90. Wilde MH, McMahon JM, Fairbanks E, et al. Feasibility

Cite this article as: Tradewell M, Pariser JJ, Nimeh T, Elliott SP; on behalf of the Neurogenic Bladder Research Group. Systematic review and practice policy statements on urinary tract infection prevention in adults with spina bifida. Transl Androl Urol 2018;7(Suppl 2):S205-S2 19. doi: 10.21037/ tau.2018.04.21 of a Web-Based Self-management Intervention for Intermittent Urinary Catheter Users With Spinal Cord Injury. J Wound Ostomy Continence Nurs 2016;43:529-38.

91. Stothers L, Nigro MK, Deegan E, et al. High user satisfaction and reduction of clinical urinary tract infections using a standardized telemedicine platform in the neurogenic bladder and spinal cord injury population. Can Urol Assoc J 2017;11:S191.

92. Dicianno BE, Lovelace J, Peele P, et al. Effectiveness of a Wellness Program for Individuals With Spina Bifida and Spinal Cord Injury Within an Integrated Delivery System. Arch Phys Med Rehabil 2016;97:1969-78.

93. Levy S, Henderson L, McAlpine C. Growing up with confidence: using telehealth to support continence selfcare deficits amongst young people with complex needs. Inform Prim Care 2014;21:113-7.

94. Smith LD. Continuity of care through nursing case management of the chronically ill child. Clin Nurse Spec CNS 1994;8:65-8.

95. Brustrom J, Thibadeau J, John L, et al. Care coordination in the spina bifida clinic setting: current practice and future directions. J Pediatr Health Care 2012;26:16-26.

96. Dunleavy MJ. The role of the nurse coordinator in spina bifida clinics. ScientificWorldJournal 2007;7:1884-9.

97. Wyndaele JJ, De Taeye N. Early intermittent selfcatheterisation after spinal cord injury. Paraplegia 1990;28:76-80.

98. Cardenas DD, Hoffman JM, Kelly E, et al. Impact of a urinary tract infection educational program in persons with spinal cord injury. J Spinal Cord Med 2004;27:47-54.

99. Eswara JR, Kielb S, Koyle MA, et al. American Urological Association Working Group on Genitourinary Congenitalism. The Recommendations of the 2015 American Urological Association Working Group on Genitourinary Congenitalism. Urology 2016;88:1-7. 